\title{
Utilization of Lithium Incorporated Mesoporous Silica for Preventing Necrosis and Increase Apoptosis in Different Cancer Cells.
}

\author{
Kamel A. Saleh*2, Sharah A. Aldulmani' ${ }^{1}$, Nasser S. Awwad ${ }^{1}$, Mohammad Y. Alfaifi², \\ Mohamed S. Hamdy ${ }^{1}$, Hala A. Ibrahium ${ }^{2 \& 3}$ \\ ${ }^{1}$ Department of Chemistry, Faculty of Science, King Khalid University, P.O. Box 9004, Abha, Saudi Arabia. \\ ${ }^{2}$ Department of Biology, Faculty of Science, King Khalid University, P.O. Box 9004, Abha, Saudi Arabia. \\ ${ }^{3}$ Department of Biology, Nuclear Materials Authority, P.O. Box 530, El Maadi, Egypt
}

*Correspondence author.

\begin{abstract}
There are many molecules used as drug carrier. TUD-1 is a newly synthesized mesoporous silica (SM) molecule possess two important features; consists of mesoporous so it is very suitable to be drug carrier in addition to that it has the ability to induce apoptosis in cancer cells. However, the effect of TUD-1 appears to act as cell death inducer, regardless of whether it is necrosis or apoptosis. Unfortunately, recent studies indicate that a proportion of cells undergo necrosis rather than apoptosis, which limits the use of TUD-1 as a secure treatment. On the other hand, lithium considered as necrosis inhibitor element. Hence, current study based on the idea of production a new Li/TUD-1 by incorporated mesoporous silica (TUD-1 type) with lithium in order to produce a new compound that has the ability to activate apoptosis by mesoporous silica (TUD-1 type) and at the same time can inhibit the activity of necrosis by lithium. Herein, lithium was incorporated in TUD-1 mesoporous silica by using sol-gel technique in one step synthesis procedure. Moreover, lithium was incorporated in TUD-1 with different loading in order to form different active sites such as isolated lithium ions, nanoparticles of $\mathrm{Li}_{2} \mathrm{O}$, and bulky crystals of $\mathrm{Li}_{2} \mathrm{O}$.

The ability of the new compounds to induce apoptosis and prevent necrosis was evaluated on three different types of cancer cell lines which are; liver HepG-2, Breast MCF-7 and colon HCT116. The obtained results show that Li/TUD-1has the ability to control necrosis and thus reduce the side effects of treatments containing silica in the case of lithium has been added to them, especially in chronic cases. This has been demonstrated by the significant increase in the IC50 value and cell viability comparing to control groups. Consequently, the idea is new, so it definitely needs more develop and test with materials that have more apoptotic impact than silica in order to induce apoptosis without induction of necrosis.
\end{abstract}

Keywords: Lithium silicate; Mesoporous silica; Necrosis; Li/TUD-1; NA-K Pump; Apoptosis.

\section{Introduction}

Invention of a new anti-cancer treatment is related to understanding the mechanism of entering the molecules into the cell and then following the pathway (s) which the treatment will activates in order to induce cells to apoptosis. Hence, molecules with high permeability or loading high-impact molecules should be selected for vectors that have the ability to enter cells easily $[1,2]$. But not every molecule that can enter cells has the ability to activate the appropriate mechanisms to kill or to keep cancer cells under control. For example, mesoporous silica nanoparticles (MSN) have the ability to enter cell by enhance permeability or retention effect [3]. These particles have gained their importance from possessing a large surface area and their pores volume [4-6]. Consequently, many vital applications that rely directly on these properties have emerged like immediate/sustained drug delivery systems, biotherapeutic agent delivery, controlled/targeted drug delivery systems [6-9]. Moreover, MSN have special role in bio-imaging applications and bioactive materials for tissue regeneration [10-12]. In addition to that, it can be considered as biocompatible materials [13]. However, the main challenge lies in the ability of silica not to induce apoptosis but its ability to activate necrosis mechanisms. While the ideal anti-cancer therapies and drug design seek to induce apoptosis without activating necrosis. The reason for that, cells undergoing apoptosis will produce apoptotic bodies and membrane blebs which 
activate the immune system [14-16]. Wherefore, mesoporous silica alone is not favored as anti-cancer treatment despite its ability to penetrate and eliminate as many cancer cells as possible, due to its ability to induce necrosis along with apoptosis. In order to fix this problem anew modified mesoporous silica molecule should be designed.

On the other hand, lithium has been tested for many years as a treatment for the mood disorders $[17,18]$; it can be deemed a safe treatment for normal cells because it protects nerve cells from necrosis by inhibiting the activity of anti-apoptosis bcl-2 gene [19-22]. In addition to modulate cytokine production, gene expression lithium can induce apoptosis and stimulates the cell division of neuroblasts in primary cultures $[22,23]$. Based on the above, it can be said that silica has a great role as a drug vector and at the same time apoptosis promoter, but its side effect is to stimulate necrosis. Whereas Lithium stimulates the cell to divide, if it fails, it prevents it from going to necrosis by inhibition of bcl-2.

In attempt to produce double sided effect and triple feature drug, lithium was incorporated onto mesoporous silica TUD-1 in order to combine the three good features (enhances apoptosis by silica, decreases necrosis by lithium in addition to use as drug delivery molecule) this study was designed. The new Li/TUD-1 that combines the qualities of silica in terms of easy entry to the cell as a carrier and urged by the cell to apoptosis, and lithium, which protects the cell from going to necrosis. This new Li/TUD-1 will be known as Li/TUD-1.

\section{Materials and Methods}

\subsection{Prepared of Li doped Silica Li/TUD-1.}

Four Li/TUD-1 samples were prepared according to a molar ratio composition of $\mathrm{SiO}_{2}: \mathrm{xLi}: 0.5 \mathrm{TEAOH}$ : 1 TEA : $11 \mathrm{H}_{2} \mathrm{O}$, where $\mathrm{x}=0.01,0.05,0.1$, and 0.2 . The prepared materials were synthesized by mixing a solution consisting of triethanolamine (TEA, 97\%, ACROS) and $\mathrm{LiNO}_{3}(99 \%$ Sigma) with tetraethyl orthosilicate (TEOS, $+98 \%$, ACROS) under vigorous stirring. After 30 minutes, tetraethyl ammonium hydroxide (TEAOH, 35\%, Aldrich) was added dropwise and the overall mixture stirred for at least two hours at room temperature. The resulting homogeneous solution/gel was aged at room temperature for 24 hours, and then dried at $371 \mathrm{~K}$ for another 24 hours. The obtained solid was ground and hydrothermally treated in a $50 \mathrm{ml}$ Teflon-lined stainless steel autoclave at $451 \mathrm{~K}$ under autogenous pressure for four hours. The obtained solid was ground again, and then calcined in static air at $873 \mathrm{~K}$ for $10 \mathrm{~h}$ applying a heating ramp rate of $1 \mathrm{~K} / \mathrm{min}$. The obtained solids were stored in a clean glass bottles and kept in dissector. The four samples were coded as $\mathrm{Li}-\mathrm{x}$, where $\mathrm{x}$ is the loading wt\% which are 1,5, 10 and 20.

\subsection{Characterizations.}

The prepared materials were characterized by several techniques to understand the structure of each sample. X-ray diffraction (XRD) measurements were carried out with Shimadzu LabX-XRD-6000 diffractometer with $C u K_{\alpha}(\lambda=1.5406 \AA)$ radiation and secondary monochromator attached with Shimadzu software with pdf-2 library for the analysis of XRD data. The data collection was carried out under ambient conditions. Moreover, FT-IR spectra of Li/TUD-1 were recorded using THERMO SCIENTIFIC, DXR FT-IR spectrometer by KBr pellet method in the wavenumber range of 4000-400 $\mathrm{cm}^{-1}$. Finally, the morphological structure of prepared materials were characterized by field emission scanning electron microscope (FE-SEM) (JSM-7500 F; JEOL-Japan) equipped with energy dispersive spectroscopy (EDS) microanalysis system.

\section{Results and Discussion}

\subsection{Characterization data}


XRD spectra of Li/TUD- 1 samples were measured within the $2 \theta$ range of $5-70^{\circ}$ are presented in Figure 1. The XRD patterns for all the samples showed one broad intensive peak at $23^{\circ}$ which can be attributed to the amorphous phase of $\mathrm{SiO}_{2}$ [F. Adam, T.S. Chew, J.J. Andas, SoleGel Sci. Technol. 59 (2011) 580583]. Moreover, no more phases were observed in $\mathrm{Li}-1$ sample as an indication for the total incorporation of $\mathrm{Li}$ ions in the silica framework. As the loading of Li increased, new peaks were observed at $23.7^{\circ}$, $24.6^{\circ}, 26.0^{\circ}, 26.6^{\circ}, 28.5^{\circ}, 37.7^{\circ}$ that. These peaks can be attributed to the formation of crystalline phases of $\mathrm{Li}_{4} \mathrm{SiO}_{4}$ and $\mathrm{Li}_{8} \mathrm{SiO}_{6}$. The crystals formation of lithium silicate different phases was also observed by Wang and co-working during the synthesis of Li-SBA-15 [Y Pan, Y. Zhang, T. Zhou, B. Louis, D. O'Hare, Q. Wang, Inorg. Chem. 2017, 56, 7821-7834]. Therefore; the XRD results showed the formation of isolated $\mathrm{Li}$ sites in $\mathrm{Li}-1$, nanoparticles of crystalline Li-silicate phases in $\mathrm{Li}-5$ and $\mathrm{Li}$ 10 samples and finally, bulky extra-framework crystals of Li-silicate different phases were detected. The formation of different dopant structure by increasing the loading of the dopant was also observed in the work of Hamdy and coworkers [M.S. Hamdy, G. Mul, Applied Catalysis B: Environmental 174 (2015) 413-420 // M.S. Hamdy, G. Mul, Catal. Sci. Technol., 2012, 2, 1894-1900].
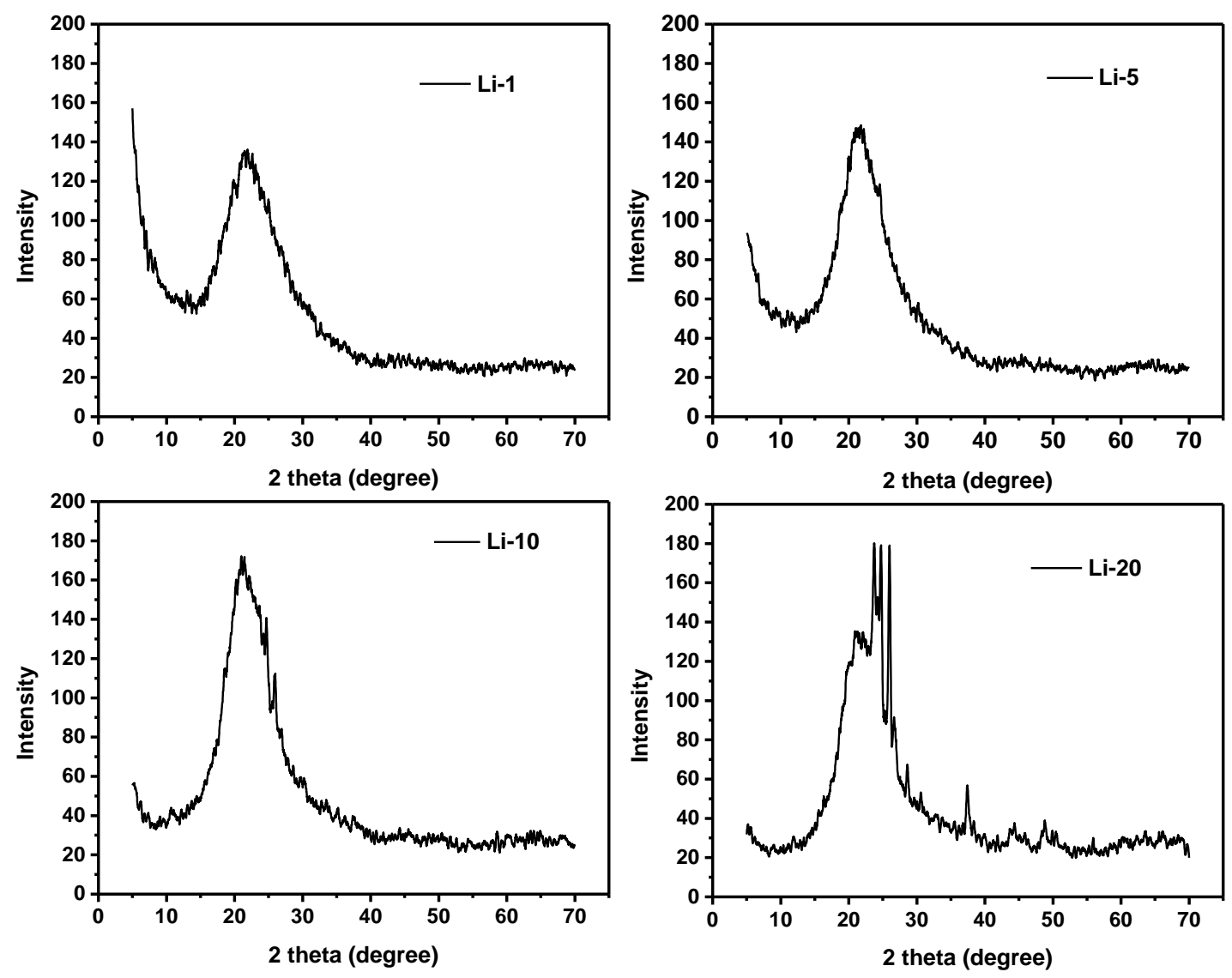

Figure 1. The X-Ray Diffraction (XRD) patterns of the prepared Li/TUD-1.

FT-IR spectra of Li/TUD-1 samples were collected at ambient conditions without further treatment (Figure 2). All the peaks are dominated with two peaks at 1054 and $804 \mathrm{~cm}^{-1}$, which can be attributed to the asymmetric stretching and symmetric modes of $\mathrm{Si}-\mathrm{O}-\mathrm{Si}$ lattice vibrations, respectively [C.T. Wang, C.L. Wu, Thin Solid Films 496 (2006) 658-664.// P.G. Su, Y.L. Sun, C.S. Wang, C.C. Lin, Sens. Actuators B 119 (2006) 483-489.]. It was established that the peak near $950 \mathrm{~cm}^{-1}$ can be attributed to the surface silanol group $(\mathrm{Si}-\mathrm{OH})$. The intensity of this peak was stronger with $\mathrm{Li}$ content, this can be explained by the formation of more $\mathrm{Si}-\mathrm{OH}$ groups due to the hydrophilicity of $\mathrm{Li}$-silicate crystals which adsorb more water molecules. These adsorbed water molecules interact with the $\mathrm{Si}-\mathrm{O}-\mathrm{Si}$ of TUD-1 to form the surface hydroxyls. Hence, the intensity of the peak became stronger [R.J. Wu, Y.L. Sun, C.C. Lin, H.W. Chen, M. Chavali, Sens. Actuators B 115 (2006) 198-204.]. Moreover, the intensity of 804 
$\mathrm{cm}^{-1}$ band was gradually decreasing with Li content, and other bands at 779, 740, and $628 \mathrm{~cm}^{-1}$ were clearly seen. These bands can be attributed to the formation of lithium silicate crystals. Finally, the obtained FT-IR results are in a good agreement with the XRD results.

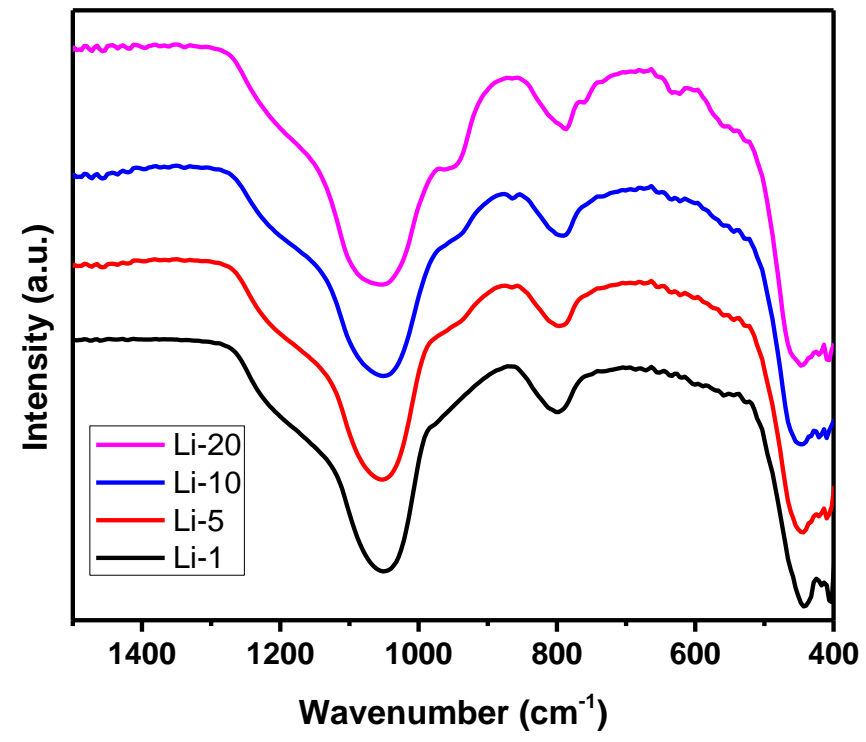

Figure 2. The FTIR spectra of the prepared Li/TUD-1 samples.

Figure 3 shows the scanning electron microscope (SEM) micrographs of Li-1 and Li-20 samples. In Li1 micrographs, only irregular smooth surface particles were detected. The surface morphological structure of Li-1 sample is typical to that of TUD-1 silica material [A.A. Alshehri, A.M. Alhanash, M. Eissa, M.S. Hamdy, Appl. Catal. A, 554 (2018) 71-79]. No extra-framework particles could be detected. However, in Li-20 sample, the micrographs show in addition to the silica particles, extra-framework of small spherical lithium silicate particles. This result is in-line with XRD and FT-IR results which all are indicated that at high loading of $\mathrm{Li}$, extra-framework of lithium silicate phase are formed.
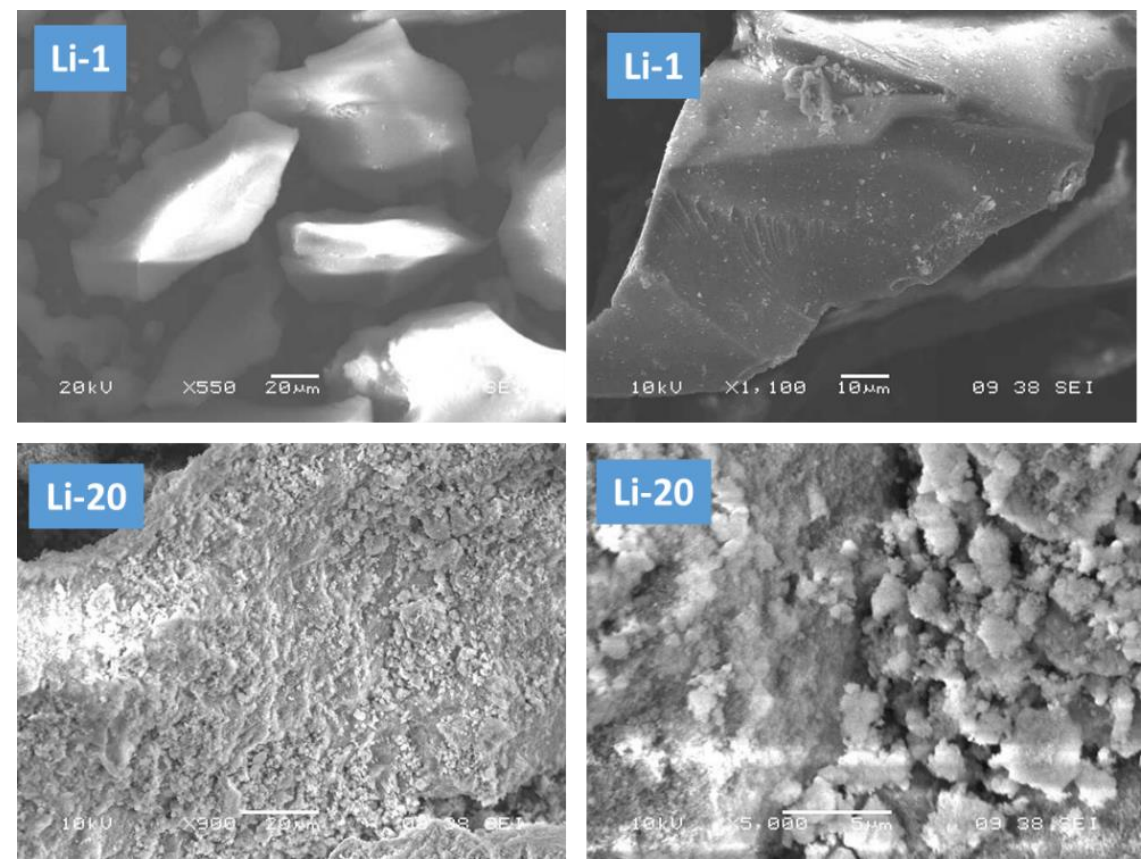
Figure 3. SEM micrographs of the prepared Li-1 and Li-20 samples.

\subsection{Antitumor Activity}

\subsection{Chemicals and drugs}

All chemicals used in this study (SulphoRhodamine-B (SRB, Tris-HCl, Tricloroacitic acid (TCA)) were purchased from Sigma Chemical Co. (St. Louis, MO, USA). While media and related products were supplemented from Gibco / Life Technologies Co, (Carlsbad, CA, USA).

\subsection{Cell culture}

Cells Human hepatic carcinoma (HEPG-2), breast (MCF-7) and colon (HCT116) used in current study were purchased from Vacsera (Giza, Egypt). In preparation to treatment cells maintained in cell culture media RPMI which is supplemented with $100 \mu \mathrm{g} / \mathrm{mL}$ streptomycin/penicillin and $10 \%$ heatinactivated (FBS) fetal bovine serum.

\subsection{Cell Viability detection}

The cytotoxicity of the elements and were tested against HEPG-2, MCF-7 and colon HCT116 tumor cell lines. Confluent $80 \%$ cells were exposed to the Li alone, TUD-1 alone and Li/TUD-1 with $(0.01,0.1,1,10,100 \mu \mathrm{g} / \mathrm{ml})$ for $72 \mathrm{~h}$. SRB evaluation test was used in order to determine the $\mathrm{IC}_{50}$ value. This value is very use full and important to evaluate the effective dose for each concentration.

Table 1: The impact of Li, TUD-1, and Li/TUD-1 Li/TUD-1 on cell viability against cancer cell lines.

\section{Cell type}

\section{Cell Viability}

\begin{tabular}{ccccccc} 
& TUD-1 & Li & \multicolumn{4}{c}{ Li/TUD-1 } \\
\hline & & & $\mathbf{1}$ & $\mathbf{5}$ & $\mathbf{1 0}$ & $\mathbf{2 0}$ \\
\hline HEPG2 & $53 \%$ & $87.9 \%$ & $84.2 \pm 1.97$ & $89.5 \pm 2.40$ & $93.42 \pm 1.44$ & $92.8 \pm 1.11$ \\
\hline MCF-7 & $56 \%$ & $61.4 \%$ & $75.9 \pm 1.54$ & $77.91 \pm 3.37$ & $80.5 \pm 3.9$ & $77.6 \pm 1.75$ \\
\hline HCT116 & $45 \%$ & $90.5 \%$ & $90.13 \pm 1.76$ & $92.15 \pm 2.46$ & $94.04 \pm 2.14$ & $92.42 \pm 1.71$
\end{tabular}



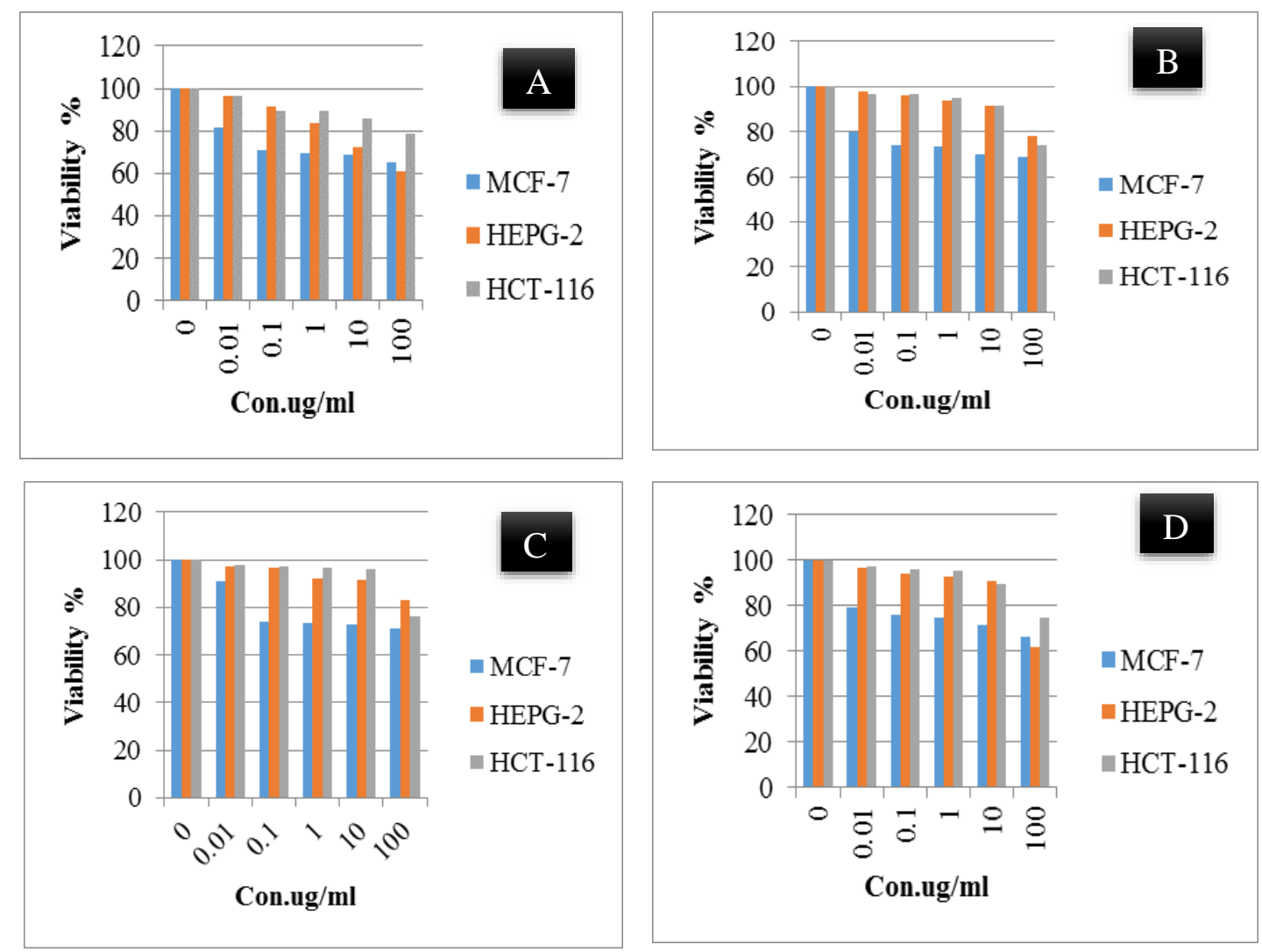

Figure 5. Cell viability curves of HepG2, MCF-7 and HCT-116 cancer cell lines after exposure to different concentrations of Li/TUD-1 for 72 hrs. Where A; Con.1, B; Con. 5, C; Con. 10 and D; Con. 20.

\subsection{IC50 detection}

Our results confirmed that the Silica (TUD-1), Li, and the (Li/TUD-1) exhibit different cytotoxicity impact against HEPG-2, MCF-7 and colon HCT116 tumor cell lines with the IC50 values $>100 \mu \mathrm{g} / \mathrm{ml}$, for TUD-1 and Li separately while the impact was $>1000$ in all cell lines treated with Li/TUD-1 for each concentration $(0.01,0.1,1,10,100 \mu \mathrm{g} / \mathrm{ml})$. Interesting point here is $\mathrm{IC}_{50}$ going to be increased parallel with the Li/TUD-1 concentration. While concentration 20 exhibited different behavior where increased instead of decreased (Table 2). Determined $\mathrm{IC}_{50}$ had been given as diagram in figure 6.

Table 2: The impact of TUD-1, Li and Li/TUD-1Li/TUD-1 as IC50 against cancer cell lines.

\begin{tabular}{cccc}
\hline Cell type & \multicolumn{3}{c}{ IC $_{50}$} \\
\cline { 2 - 4 } & TUD-1 & Li & $\begin{array}{c}\text { Li/TUD-1/ for con. } \\
(1,5,10,20)\end{array}$ \\
\hline HEPG2 & $>100$ & $>100$ & $>1000$ \\
\hline MCF-7 & $>100$ & $>100$ & $>1000$ \\
\hline HCT116 & $>100$ & $>100$ & $>1000$ \\
\hline
\end{tabular}



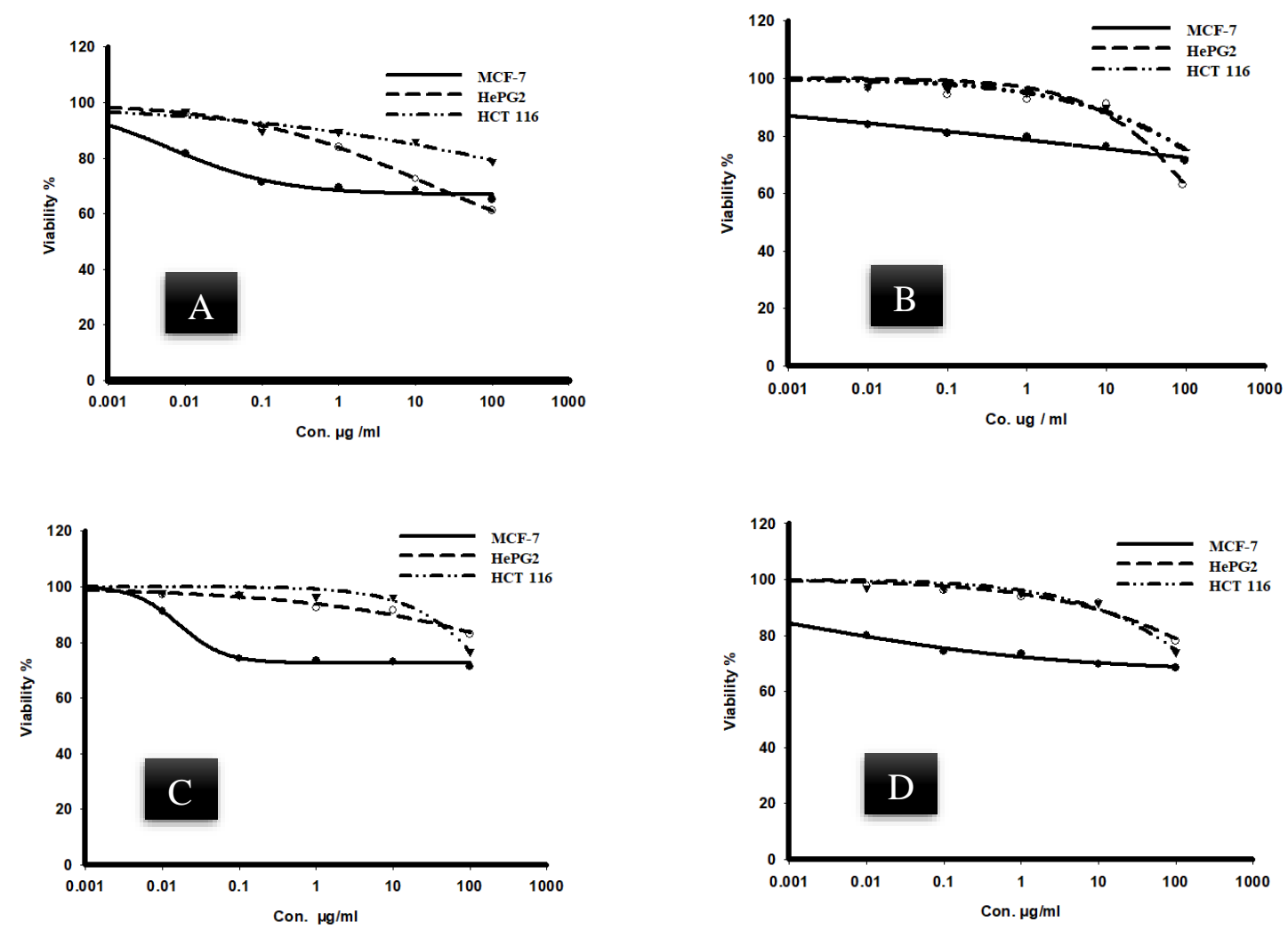

Figure 6. The response curves of different cancer cell lines HepG2, MCF-7 and HCT-116 after exposure to different concentrations of Li/TUD-1 for 72 hrs. Where A; Con.1, B; Con. 5, C; Con. 10 and D; Con. 20.

\subsection{Statistical Analysis}

The data were analyzed using Sigma Plot version 12.0.

\subsection{Apoptosis induction}

Previous results showed that there is a significant improvement in the event of the use of Li/TUD-1 (Si$\mathrm{Li}$ ) comparing to use elements alone. But the difficulty lies in identifying the mechanism that caused it. Therefore, in attempt to confirm the mechanism of action, cells were stained with Acridine Orange and Ethidium Bromide (1:1) to detect apoptotic bodies which are considered as an important indicator of the impact mechanism (Fig 6). Consequently, to explore the mechanism of action, it was necessary to survey apoptotic and necrotic indicators (apoptotic bodies, plasma membrane alteration, condensed nucleus...etc.) appear on different cancer cell lines after treated with IC50. On the other hand, the protective role that had been played by the Li/TUD-1 (Li/TUD-1) in preventing of necrosis and allow of apoptosis had been evaluated by count the apoptotic and the necrotic cell per 100 cell. Consequently, HepG-2, MCF-7 and HCT-116 cancer cell lines were treated with IC50 concentrations determined before. Results showed that Si-Li Li/TUD-1 has moderately decreased necrosis induction on all cancer cell lines, while the apoptotic impact was increased almost 2 times comparing to $\mathrm{Si}$ or Li separately (Table 3). 
Table 3: Apoptosis induction ratio versus necrosis in different cancer cell lines per 100 cell after treated with determined $\mathrm{IC}_{50}$ dose of TUD-1, Li and Li/TUD-1Li/TUD-1.

\begin{tabular}{|c|c|c|c|c|c|c|}
\hline \multirow{2}{*}{ Cell type } & \multicolumn{3}{|c|}{ Apoptosis/100 cell } & \multicolumn{3}{c|}{ Necrosis/ 100 cell } \\
\cline { 2 - 7 } & TUD-1 & Li & Li/TUD-1 & TUD-1 & Li & TUD-1 \\
\hline HEPG2 & 16 & 28 & 43 & 25 & 16 & 12 \\
\hline MCF-7 & 18 & 22 & 55 & 33 & 12 & 8 \\
\hline HCT116 & 14 & 25 & 52 & 30 & 15 & 10 \\
\hline
\end{tabular}
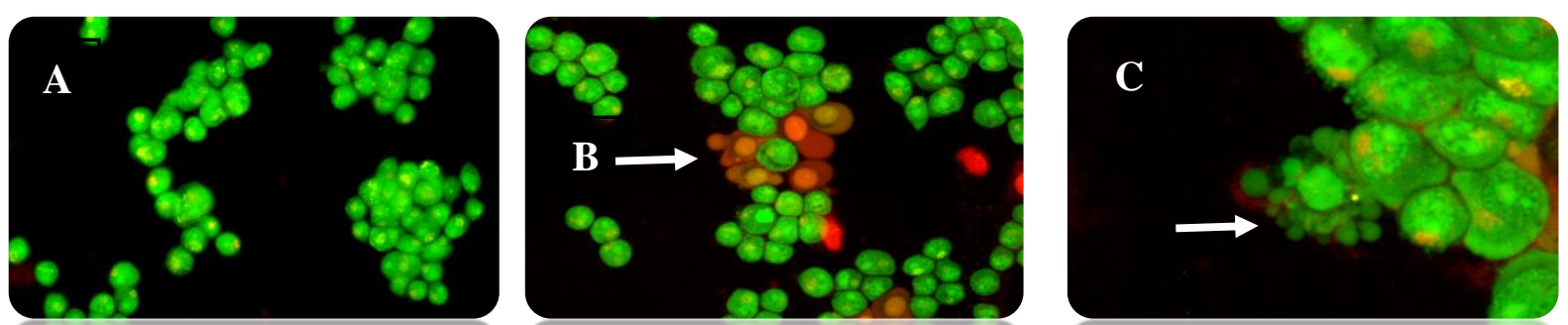

Figure 7. Detection of necrosis and apoptosis indicators presented on HepG-2, MCF-7 and HCT-116 cancer cell lines after treated with $\mathrm{IC}_{50}$ concentration of different samples where $\mathrm{A}$ : Control group, $\mathrm{B}$ : Necrotic cells, C: Apoptotic bodies presented in cells. All cells stained with (AO+EtBr), detected by Olympus fluorescent microscope after 72 hrs.

\section{Discussion}


Cellular death is controlled by two different pathways, apoptosis and necrosis [24]. Both of these mechanisms was acceptable as anticancer [25, 26]. However, recent studies confirmed that necrosis can be activate under many reasons like injury, infection, infraction, toxin, inflammation and the interesting cause is cancer itself [27]. In the light of these recent observations, very important question was present; why cancer cells choose necrosis to death instead of apoptosis. This question remained no answer until many observations confirmed that the cancer cells trying to keep as a way as possible from what may cause the activation of the immune system [28]. For this reason, apoptosis considered as the most favorite pathway to remove cancer cells under immune system control [28]. On the other hand, necrosis not only can't activate immune system but also leads inflammation [29]. This challenge leads us to understand the mechanisms that can be followed in order to activate apoptosis but not necrosis. In this study, a novel molecule (Li/TUD-1) had been invented in order to induce apoptosis and inhibit necrosis at once. To achieve this goal, two molecules were combined: the first one (TUD-1) is known as good carrier in addition to its ability to stimulate apoptosis but also causes necrosis. While the second one ( $\mathrm{Li}$ ) known for its ability to prevent necrosis. Consequently, the difference between apoptosis and necrosis in all samples before and after treated with the combined had been monitored in order to be sure that the new Li/TUD-1 had gain the requested ability. Results revealed that; the lowest concentration had the lowest activity this may due to the mesoporous TUD-1 worked as a good carrier but the small Li concentration had no impact to prevent necrosis, While the moderate concentrations (510) had clear effect in inducing apoptosis (Actually not significant comparing to control group) but the good point here the ability of this concentration to prevent necrosis significantly. On the other hand, the effect returned to weaken and become negligible at a concentration of 20 as - apparently - the pores of silica had been filled and no more space for more loading. This clearly demonstrates several important things like silica has a certain capacity as a carrier, and the increase in it does not increase its effect, but negatively affects the result, as the apoptosis begins disappearance gradually whereas the emergence of the necrosis. The cause of the emergence of necrosis may be due to the accumulation of excess amounts of the carrier's capacity in the media and around cells which prevented the oxygen absorption. Consequently, cells went to death under the induction of hypoxia but not under the impact of Li/TUD$1 \mathrm{Li} / \mathrm{TUD}-1$. The interesting point is the ability of the Li/TUD-1 to induce apoptosis and prevent necrosis in moderate concentrations. This indicates that silica as a vector is still active, and it facilitates the entry of lithium into the cells, while -at the same time- it does not affect its anti-necrosis activity. It seems that lithium leaves the pores to begin its work in inhibiting the work of bcl 2 and thus prevents or reduces the appearance of the apoptosis to a large extent. But there are some unhealthy signs where $\mathrm{IC}_{50}$ value went to be high, indicating that a part of the cells moved from necrosis to programmed cell death, so this new molecule has reduced necrosis and this is good but it did not stop the cells from dividing, which led to increase in the proportion of cells that have not died either by necrosis or programmed death.

In fact, the molecule was worked within the road map had been set for it, significantly reducing the proportion of necrosis, but in the opposite it appears to have reduced the cytotoxicity of TUD-1 as the cell death rate (IC50) has increased rather than decreased, so this new molecule has actually been prevented the occurrence of necrosis but also reduced the activity of silica catalyst for apoptosis.

\section{Conclusions}

Our goal in this research was to find a mechanism to reduce the necrosis while induce apoptosis in order to stimulate immune system later on. This study reveals that we have succeeded in molecule design, but the impact of the new Li/TUD-1 on different cancer cells remain in the beginning and must be discussed extensively in vitro as well as in vivo.

\section{Acknowledgments}


The authors extend their appreciation to the Deanship of Scientific Research at King Khalid University for funding this work through General Research Project under grant number (R.G.P 140).

\section{References}

(1) 1. Futaki, S., Membrane permeable peptide vectors: chemistry and functional design for the therapeutic applications. Adv Drug Deliv Rev, 2008. 60(4-5): p. 447.

(2) 2. Nakase, I. and S. Futaki, [Development of membrane-permeable peptide vectors and their internalization mechanisms]. Seikagaku, 2009. 81(11): p. 992-5.

(3) 3. Rosenholm, J.M., et al., Cancer-cell-specific induction of apoptosis using mesoporous silica nanoparticles as drug-delivery vectors. Small, 2010. 6(11): p. 1234-41.

(4) 4. Huang, L., et al., Direct surface grafting of mesoporous silica nanoparticles with phospholipid choline-containing copolymers through chain transfer free radical polymerization and their controlled drug delivery. J Colloid Interface Sci, 2017. 508: p. 396-404.

(5) 5. Santha Moorthy, M., et al., Synthesis of surface capped mesoporous silica nanoparticles for pH-stimuli responsive drug delivery applications. Medchemcomm, 2017. 8(9): p. 1797-1805.

(6) 6. Ahmadi, E., et al., Synthesis and surface modification of mesoporous silica nanoparticles and its application as carriers for sustained drug delivery. Drug Deliv, 2014. 21(3): p. 164-72.

(7) 7. Mao, L., et al., Photo-induced surface grafting of phosphorylcholine containing copolymers onto mesoporous silica nanoparticles for controlled drug delivery. Mater Sci Eng C Mater Biol Appl, 2017. 79: p. 596-604.

(8) 8. Sen Karaman, D., S. Manner, and J.M. Rosenholm, Mesoporous silica nanoparticles as diagnostic and therapeutic tools: how can they combat bacterial infection? Ther Deliv, 2018. 9(4): p. 241-244.

(9) 9. Zhang, Q., et al., A surface-grafted ligand functionalization strategy for coordinate binding of doxorubicin at surface of PEGylated mesoporous silica nanoparticles: Toward $\mathrm{pH}$-responsive drug delivery. Colloids Surf B Biointerfaces, 2017. 149: p. 138-145.

(10) $10 . \quad \mathrm{Li}, \mathrm{H}$. , et al., In vivo near infrared fluorescence imaging and dynamic quantification of pancreatic metastatic tumors using folic acid conjugated biodegradable mesoporous silica nanoparticles. Nanomedicine, 2018. 14(6): p. 1867-1877.

(11) 11. Sun, Q., et al., Theranostic Nanoplatform: Triple-Modal Imaging-Guided Synergistic Cancer Therapy Based on Liposome-Conjugated Mesoporous Silica Nanoparticles. ACS Appl Mater Interfaces, 2018. 10(2): p. 1963-1975.

(12) $12 . \quad$ Rosenholm, J.M., et al., Mesoporous silica nanoparticles in tissue engineering--a perspective. Nanomedicine (Lond), 2016. 11(4): p. 391-402.

(13) 13. Sahoo, B., et al., Biocompatible mesoporous silica-coated superparamagnetic manganese ferrite nanoparticles for targeted drug delivery and MR imaging applications. J Colloid Interface Sci, 2014. 431: p. 31-41.

(14) $14 . \quad$ Patel, V.A., et al., The affirmative response of the innate immune system to apoptotic cells. Autoimmunity, 2007. 40(4): p. 274-80.

(15) $15 . \quad$ Gregory, C.D., CD14-dependent clearance of apoptotic cells: relevance to the immune system. Curr Opin Immunol, 2000. 12(1): p. 27-34.

(16) $16 . \quad$ Zhang, S., et al., A novel polysaccharide from Ganoderma atrum exerts antitumor activity by activating mitochondria-mediated apoptotic pathway and boosting the immune system. J Agric Food Chem, 2014. 62(7): p. 1581-9.

(17) $17 . \quad$ Tondo, L. and R.J. Baldessarini, Antisuicidal Effects in Mood Disorders: Are They Unique to Lithium? Pharmacopsychiatry, 2018. 51(5): p. 177-188.

(18) $18 . \quad B a u e r$, M., 70 Years of Research and 50 Years of Lithium Clinics: From Serendipity to Gold Standard in Mood Disorders. Pharmacopsychiatry, 2018. 51(5): p. 165.

(19) $19 . \quad$ Brown, E.E., et al., Psychiatric benefits of lithium in water supplies may be due to protection from the neurotoxicity of lead exposure. Med Hypotheses, 2018. 115: p. 94-102. 
(20) 20. Odeya, D., A. Galila, and T. Lilah, The observed alteration in BCL2 expression following lithium treatment is influenced by the choice of normalization method. Sci Rep, 2018. 8(1): p. 6399.

(21) 21. Yao, R., et al., Lithium chloride inhibits cell survival, overcomes drug resistance, and triggers apoptosis in multiple myeloma via activation of the Wnt/beta-catenin pathway. Am J Transl Res, 2018. 10(8): p. 2610-2618.

(22) 22. Wang, F., et al., [Depressant effect of Lithium on apoptosis of nerve cells of adult rats after spinal cord injury]. Zhongguo Gu Shang, 2018. 31(4): p. 379-385.

(23) 23. Ricken, R., et al., Cytokine serum levels remain unchanged during lithium augmentation of antidepressants in major depression. J Psychiatr Res, 2018. 96: p. 203-208.

(24) 24. Joshi, G.N. and D.A. Knecht, Silica phagocytosis causes apoptosis and necrosis by different temporal and molecular pathways in alveolar macrophages. Apoptosis, 2013. 18(3): p. 271-85.

(25) 25. Al-Sadoon, M.K., et al., Induction of apoptosis and growth arrest in human breast carcinoma cells by a snake (Walterinnesia aegyptia) venom combined with silica nanoparticles: crosstalk between Bcl2 and caspase 3. Cell Physiol Biochem, 2012. 30(3): p. 653-65.

(26) $26 . \quad$ Gambelli, F., et al., Phosphorylation of tumor necrosis factor receptor 1 (p55) protects macrophages from silica-induced apoptosis. J Biol Chem, 2004. 279(3): p. 2020-9.

(27) $27 . \quad$ Sasaki, M., et al., Tumor necrosis factor-alpha induces expression of C/EBP-beta in primary afferent neurons following nerve injury. Neuroscience, 2014. 279: p. 1-9.

(28) 28. Awad, A.S., et al., Macrophage-derived tumor necrosis factor-alpha mediates diabetic renal injury. Kidney Int, 2015. 88(4): p. 722-33.

(29) 29. Evashwick-Rogler, T.W., et al., Inhibiting tumor necrosis factor-alpha at time of induced intervertebral disc injury limits long-term pain and degeneration in a rat model. JOR Spine, 2018. 1(2). 